\author{
ARTIGO \\ DOI: https://doi.org/10.22481/praxis.v14i29.4113
}

\title{
LOS SENTIDOS DE LA CULTURA ESCRITA EN LA CREAR (1973-1975)
}

\author{
SENSES OF THE WRITTEN CULTURE IN THE CREAR (1973-1975)
}

\author{
OS SENTIDOS DA CULTURA ESCRITA NA CREAR (1973-1975)
}

\author{
Mariana Tosolini \\ Universidad Nacional de Córdoba - Argentina
}

\begin{abstract}
Resumo: Este artigo trata sobre os acessos à cultura escrita que é testada a partir das campanhas de alfabetização. Essas políticas foram amplamente divulgadas, principalmente dos anos 1940. Muitos deles foram guiados pelas diretrizes das organizações internacionais a partir de uma concepção de conhecimento que Freire chamou de "digestivo". As campanhas de alfabetização nos permitem dar uma olhada política na cultura escrita. E, por isso, ele nos pergunta: o que as propostas maciças de alfabetização implicam em termos de como as subjetividades são construídas em relação à cultura escrita? Nesta apresentação, consideramos os resultados da investigação para obter o grau de médico. Analisamos os significados da cultura escrita na Campanha para a Reativação Educativa de Adultos para Reconstrução (CREAR) desenvolvida na Argentina entre 1973 e 1975. Esta experiência apresenta notas distintivas sobre a proposta de alfabetização, uma vez que disputou os sentidos hegemônicos. É interessante analisar como o acesso à cultura escrita ligada a outras práticas sociais foi configurado naquela experiência educacional. Em particular, consideramos como a proposta de alfabetização é inserida nas práticas de organização comunitária. Para isso contamos com documentos da campanha e os depoimentos dos professores de alfabetização. A partir de sua análise, contribuímos com reflexões sobre os quadros das políticas educacionais e da educação popular nos anos 1970, mostrando como nesta convergência uma proposta política pedagógica foi projetada para promover o acesso à cultura alfabetizada nos processos de organização social. Em primeiro lugar, apresentamos algumas considerações teóricas que apoiam a análise das políticas de cultura e alfabetização escritas. Em seguida, analisamos CREAR como uma política de alfabetização, com foco nas concepções que estruturaram a proposta. Em terceiro lugar, abordamos a proposta de alfabetização e as decisões metodológicas. Finalmente, apresentamos algumas reflexões como um fechamento.
\end{abstract}

Palavras chaves: Cultura escrita. Grupos operacionais. Concientização. Práctica social

\begin{abstract}
Abstrac: This paper discuss about the accesses to the written culture that is tried from the literacy campaigns. These policies were widely disseminated, mainly from the 1940s. Many of them were guided by the guidelines of international organizations from a conception of knowledge that Freire called "digestive". Literacy campaigns allow us to take a political look at written culture. And for that reason, he asks us: what do the massive literacy proposals imply in terms of how subjectivities are constructed in relation to written culture? In this presentation we put part of the results of the investigation into consideration to obtain the degree of doctor. We analyzed the meanings of the culture written in the Campaign for the Educational Reactivation of Adults for Reconstruction (CREAR) developed in Argentina between 1973 and 1975. This experience presents distinctive notes regarding the literacy proposal since it disputed the hegemonic senses. It is interesting to analyze how the access to written culture linked to other social practices was configured in that educational
\end{abstract}


experience. In particular, we consider how the literacy proposal is inserted into community organization practices. For this we rely on documents of the campaign and the testimonies of the literacy teachers. From their analysis we contribute reflections on the frameworks of educational policies and popular education in the 1970s, showing how in this convergence a pedagogical political proposal was designed to promote access to literate culture in processes of social. First, we present some theoretical considerations that support the analysis of written culture and literacy policies. Then we analyze CREAR as a literacy policy, focusing on the conceptions that structured the proposal. Third, we address the literacy proposal and the methodological decisions. Finally we present some reflections as a closing.

Keywords: Written culture. Operational groups. Awareness. Social practice

Resumen: Este artículo trata sobre los accesos a la cultura escrita que se pretende desde las campañas de alfabetización. Estas políticas tuvieron amplia difusión, fundamentalmente a partir de la década del 1940. Muchas de ellas estuvieron orientadas por los lineamientos de los Organismos internacionales desde una concepción del conocimiento que Freire denominó "digestiva". Las campañas de alfabetización nos permiten realizar una mirada política sobre la cultura escrita. Y por ello nos interpela ¿qué implican las propuestas masivas de alfabetización en cuanto a cómo se construyen las subjetividades con relación a la cultura escrita? En esta presentación ponemos a consideración parte de los resultados de la investigación para obtener el grado de doctora ${ }^{1}$. Analizamos qué sentidos tuvo la cultura escrita en la Campaña de Reactivación Educativa de Adultos para la Reconstrucción (CREAR) desarrollada en Argentina entre 1973 y 1975. Esta experiencia presenta notas distintivas en cuanto a la propuesta de alfabetización ya que disputó los sentidos hegemónicos. Interesa analizar cómo se configuró en esa experiencia educativa, el acceso a la cultura escrita vinculado a otras prácticas sociales. Particularmente, consideramos cómo se inserta la propuesta de alfabetización en prácticas de organización comunitaria. Para ello nos basamos en documentos de la campaña y los testimonios de los alfabetizadores. A partir de sus análisis aportamos reflexiones sobre los entramados de las políticas educativas y la educación popular en los años 70, mostrando cómo en esta convergencia se construyó una propuesta político pedagógica destinada a promover el acceso a la cultura letrada en procesos de organización social. En primer lugar, presentamos algunas consideraciones teóricas que sostienen el análisis de la cultura escrita y las políticas de alfabetización. Luego analizamos la CREAR como política de alfabetización, focalizando en las concepciones que estructuraron la propuesta. En tercer lugar, abordamos la propuesta de alfabetización y las decisiones metodológicas. Finalmente presentamos algunas reflexiones a modo de cierre.

Palabras claves: Cultura escrita. Grupos operativos. Concientización. Práctica social

\section{La cultura escrita: dimensión política}

Las relaciones con la cultura escrita son parte de un proceso de socialización y se da mediada por relaciones sociales que funcionan como organizadores de la experiencia de los

\footnotetext{
${ }^{1}$ Este trabajo es parte de la Investigación para obtener el grado de doctor en Estudios Sociales de América Latina. Centro de Estudios Avanzados; Universidad Nacional de Córdoba. Beca SeCyT. Años 2010-2005. Directora: Dra. María del Carmen Lorenzatti. Co-directora: Dra. Liliana Vanella.

Este estudio se enmarca en el Proyecto "Educación de jóvenes y adultos: sujetos, conocimientos y procesos de formación docente" con lugar de trabajo en el Área Educación del Centro de Investigaciones de la FFyH. Aprobado y Financiado por Secyt UNC. Res. No 313/2016. Y el proyecto "Prácticas educativas con jóvenes y adultos: políticas, sujetos y conocimiento", con lugar de trabajo en el Área Educación del Centro de Investigaciones de la FFyH. Aprobado y Financiado por FONCYT. PICT-2016-2081. Res. N 285/2017. Ambos proyectos dirigidos por la Dra. María del Carmen Lorenzatti.
} 
sujetos. De acuerdo a lo planteado por Kalman, el aprendizaje de la escritura y la lectura son sociales porque requieren aprender sistemas de usos que son construcciones sociales e históricas (2003).

Tal como señala Lorenzatti (2009), las prácticas sociales implican acciones de los sujetos con la cultura escrita y se enmarcan en prácticas sociales más amplias. Esta manera de entender la alfabetización, que la autora refiere como literacidad, permite conocer de qué manera los sujetos usan y se apropian de la cultura escrita en función de sus necesidades, intereses y motivaciones. Para esto se relacionan con diferentes personas, exponen sus puntos de vista, se defienden, argumentan, cuestionan, pelean. Se trata de prácticas sociales que incluyen actividades, acciones y propósitos particulares que implican relaciones sociales e interacciones con objetos escritos en el marco de relaciones de poder.

Rockwell (1992) advierte sobre la importancia de reconstruir e interpretar los significados de una práctica social y los usos sociales de la lengua escrita analizándolos en diferentes contextos culturales y las situaciones sociales donde se generan esas prácticas. Estas prácticas están insertas en relaciones sociales donde las contradicciones son constitutivas de esas prácticas.

En esa perspectiva Kalman, sostiene que

[...] la alfabetización tiene que entenderse como algo mucho más que la simple iniciación a las letras: es la apropiación de prácticas comunicativas mediadas por la escritura; es un proceso que abarca la apropiación del sistema de escritura, pero no se limita a ella. La alfabetización implica necesariamente los usos de la lectura y la escritura en contextos específicos; es la participación en eventos comunicativos donde leer y escribir son parte de la actividad comunicativa. (KALMAN, 2000, p.5).

$\mathrm{Si}$ bien existen diversas conceptualizaciones acerca de alfabetización nos detendremos en lo que significa entender la alfabetización como una práctica social.

Un punto de partida ineludible es el concepto de alfabetización que propone Paulo Freire. Particularmente sus ideas pedagógicas expresadas en "La educación como práctica de la libertad" publicada en 1965 y "Pedagogía del Oprimido" publicado en 1970. En estas obras, el educador brasilero Paulo Freire sostiene que la educación es un acto político y la alfabetización de adultos es una acción cultural para la libertad.

Desde finales de los '60, el autor ofrece herramientas conceptuales para romper con la visión neutra de la educación. Sostiene que el hombre se encuentra en permanente relación con el mundo que lo rodea, y por lo tanto es sujeto de su acción en el mundo a través de la construcción de su conocimiento. 
Lorenzatti (2009) señala que uno de los aportes de Freire es su mirada sobre la importancia del contexto para comprender las acciones de los sujetos y las interrelaciones entre los sujetos en la construcción del conocimiento.

La educación y alfabetización como acto político que plantea Freire en esa época, tiene el sentido de lo político como expresión total de la vida. Es importante, entonces entender la amplitud de este concepto en Freire para no reducir su significado a la codificación y por lo tanto, se interprete como opuesta a práctica social.

Si bien al concepto de alfabetización como práctica social lo introducen los Nuevos Estudios de Literacidad, la perspectiva de Freire se podría asimilar al de práctica social. En este sentido, Street (Kalman, 2008) habla de un modelo autónomo y de un modelo ideológico. El autor identifica las campañas de alfabetización con un modelo autónomo en tanto implican la concepción de la lectura y la escritura como actividades aisladas de otras prácticas sociales. El otro modelo que propone Street, el modelo ideológico, donde la alfabetización es considerada una práctica social y de esa manera rebasa una cuestión técnica para comprender lo que hacemos con la lectura y escritura. En tal sentido, Kalman señala que

[...] los eventos de la cultura escrita ocurren en escenarios institucionales y sociales específicos, en el contexto de las relaciones de poder e involucran la circulación e distintas tradiciones discursivas. (KALMAN, 2008, p.7)

El modelo ideológico nos permite establecer y situar la importancia del contexto en la definición de las campañas. En este sentido, creemos que configura un puente entre lo que propone Freire sobre la alfabetización como práctica política y la alfabetización como práctica social de los Nuevos Estudios de Literacidad. Es precisamente desde ese modelo que nos interesa analizar la CREAR en una dinámica histórica social.

La CREAR fue una experiencia educativa del pasado reciente que propuso una forma diferente de implementar las políticas y de entender la tarea educativa del estado. Tal como planteaba Gramsci (2011) interesa poner en discusión ¿Qué niveles de civilización contribuye a crear el estado con las políticas educativas que propone? En ese marco, las políticas de alfabetización son una forma de regular el acceso a los bienes culturales de la sociedad, fundamentalmente de los sujetos que han padecido múltiples exclusiones. Implican por ello la regulación de los modos de transmisión y apropiación de saberes, los que a su vez implican relaciones sociales donde se distribuyen y apropian esos saberes. En tal sentido, las políticas de alfabetización constituyen modos de intervención de los estados en la educación de adultos y son una forma de construcción de subjetividades. Particularmente es importante analizar las políticas de alfabetización hacia los adultos porque ha sido históricamente una educación 
dirigida a los grupos subalternos, y entonces es oportuno preguntarse ¿cómo son interpelados por el estado?

Ante este interrogante una categoría que cobra relevancia es la de hegemonía. Si la hegemonía tal como plantea Raymond Williams y Gramsci configuran formas educativas, el estudio de las políticas de alfabetización permite analizar cómo a través de las formas de constituir sujetos se construye un sentido de realidad.

Desde esa perspectiva, es necesario comprender las políticas de alfabetización en sus condiciones de producción. Esto implica analizarlas en la dinámica histórico social en la que son producidas. Una pregunta que articula estos sentidos es ¿cómo se construye el orden? Y las respuestas giran en torno a las luchas histórico sociales por establecer determinados valores, concepciones del mundo y relaciones sociales sobre los cuales descansan las reglas de existencia de los sujetos. En esas luchas se definen los modelos de sujetos y a ella están íntimamente ligados los modelos culturales y educativos. En esa dinámica se juegan las propuestas de alfabetización como forma de legitimación de los proyectos en disputas. ¿Cómo se sostiene un proyecto hegemónico? Nuevamente aquí, nos interpelan los sentidos sociales de las políticas de alfabetización, ya que ellas implican propuestas de formación de sujetos para la participación en la producción y en las formas políticas y ciudadanas.

Si la alfabetización es parte de un proyecto político, ese proyecto político se concreta en el sostenimiento de un orden social determinado o, en la preparación para otro modelo social, es decir, para el cambio social.

Por ello, entendemos que la alfabetización es una práctica social compleja, expresión de la totalidad social de la cual forma parte y de sus contradicciones (Puiggrós, 2005). Es decir, es una práctica donde convergen sujetos, políticas, materiales educativos y por ello requiere ser comprendida como expresión de una dinámica histórico- social.

Un concepto central para entender los contextos de alfabetización es el de totalidad social. Esta categoría que deviene del marxismo es retomada por Adriana Puiggrós y el equipo del proyecto "Alternativas Pedagógicas y prospectiva educativa en América Latina" $(\mathrm{APPEAL})^{2}$ para analizar la sociedad como un complejo dinámico, como una articulación de procesos que posibilita entender las relaciones entre los procesos educativos y sus condiciones de producción histórico-sociales (Puiggrós, 2005).

En este sentido, la autora señala que la educación - y la alfabetización -es expresión de procesos sociales y no algo exterior o accesorio a ellos. Es decir, que las condiciones de

\footnotetext{
${ }^{2}$ Retomamos construcciones conceptuales del equipo de APPEAL desde sus trabajos publicados en 1988, tanto de los miembros de Buenos Aires, como los de México.
} 
producción son internas y no externas a las experiencias educativas. Esto nos permite visualizar la alfabetización como expresión de la totalidad social, que se vincula con las relaciones sociales de producción, las luchas políticas, sociales y culturales

Las campañas de alfabetización de adultos han estado orientadas por distintas concepciones acerca de lo que implican los vínculos con la cultura escrita. En tal sentido, la definición de alfabetización se configura históricamente y está atravesada por las luchas ideológicas por delimitar sus sentidos. Por ello, estar alfabetizado es una construcción histórica y social que está relacionada a otras definiciones políticas, ideológicas y culturales.

Las campañas de alfabetización se constituyeron en América Latina en una de las formas privilegiadas de intervención de los estados en la educación de adultos. Como toda propuesta de alfabetización encierra una construcción pedagógica. La construcción pedagógica de las campañas de alfabetización implica contemplar además los sujetos sociales, el contenido, los métodos y los materiales educativos. Precisamente la complejidad de los procesos de alfabetización supone considerar las propuestas de alfabetización como construcciones donde se ponen en juego determinados vínculos con la cultura escrita.

La alfabetización en el periodo de los años 60 y 70 podría considerarse como una de las formas que asumen algunos sectores para aportar al desarrollo de la conciencia del pueblo. La alfabetización de adultos en esa perspectiva constituye un espacio propicio para transmitir determinados saberes en los sectores "pobres" y por ello se considera clave para superar el "subdesarrollo" (Devés Valdés, 2008).

De este modo, tal como plantean Peresson, Cendales y Mariño (1983) la alfabetización era considerada como una exigencia de la cultura y en este marco, nos interesa profundizar cómo se concibe la alfabetización en los procesos de cambio social.

\section{La CREAR como política de alfabetización}

La CREAR fue una propuesta político - pedagógica que se da en un contexto de radicalización política y de lucha entre los sectores del peronismo por establecer una nueva hegemonía. Esta construcción implicaba la intencionalidad de promover nuevas prácticas en el marco de procesos de institucionalización en distintos planos: económico, social, político, cultural y educativo.

La CREAR expresó como política pública, un posicionamiento sobre los modelos de desarrollo que se debatieron después de la segunda guerra mundial. En tal sentido, los modelos de desarrollo que se desplegaron en la década del '60 estuvieron sostenidos desde 
distintas concepciones. Por un lado, las que postularon las políticas desarrollistas y, por el otro, las concepciones críticas. Estas últimas estuvieron orientadas a un modelo socialista que emerge iluminado por la revolución cubana y luego la teoría de la dependencia, que realizaron una mirada crítica de los fundamentos del desarrollismo.

En ese panorama, la educación de los trabajadores y los dirigentes se configuró como un espacio complejo y problemático y por ello cuidado y pensado desde las políticas públicas. En esa dinámica, la educación del adulto se centró desde el marco político de la CREAR como la formación de los "trabajadores y marginados" y su participación en el proyecto nacional. En esa dinámica, se posicionó frente a estos procesos y construyó formas de intervención destinadas a superar la mirada del capital humano, sosteniendo la formación del pueblo como sujeto político.

En el marco de las apuestas de los distintos sectores para hacer prevalecer su mirada sobre el desarrollo, el pueblo emerge como sujeto social clave. Tal como señala Devés Valdés (2008), en algunos casos la consideración hacia el "pueblo" y lo popular" se sitúa desde una mirada romántica. Pero a partir de la propuesta de alfabetización que promovió la revolución cubana se instala el pueblo como centro de los discursos y las prácticas. Se construye un discurso donde la pobreza, la organización del pueblo y su lugar como educador (Pineau, 2008), se promovió a través de las acciones colectivas, fundamentalmente en la participación como "comunidad organizada" que se configuran en la institucionalización de prácticas educativas comunitarias.

Estas prácticas se hallaban vinculadas a procesos de ruptura del orden social. Es por ello que es posible advertir que el cambio social, como un hecho/anhelo se instala como una estructura de sentimiento (Williams, 2009) que movilizaba los proyectos de organización social y que fue acompañado por las proyecciones educativas y el compromiso intelectual y artístico. Encontramos que el cambio social se construye en la propuesta de la CREAR como revolución y por ello esa concepción articuló una serie de planteos sobre las condiciones para producir la revolución. Más precisamente, el cambio social se vincula a otro tópico de los 60: la conciencia (Devés Valdés, 2008). La conciencia y los procesos de alfabetización son construidos por Paulo Freire como caras de una misma moneda, es decir- la alfabetización- es al mismo tiempo un proceso de objetivación de condiciones de dominación y por lo tanto una herramienta para la concientización. Desde esta perspectiva, algunos puntos claves sobre la propuesta de alfabetización de la CREAR y cómo se propuso construir formas de conciencia en los trabajadores y en las comunidades se expresaron en el anclaje comunitario de los procesos de alfabetización. 
Es posible apreciar en la propuesta pedagógica de la campaña la presencia de elementos ideológicos que abonan la organización del pueblo: la militancia en los barrios, el proyecto de descolonización cultural a través de "rescate de la cultura del pueblo", las formas de concientización a partir de diversas tareas en los barrios, la impronta de la educación popular y la admiración por la experiencia cubana y después la experiencia chilena.

En ese escenario que se configura como concepción de mundo, el analfabetismo se presenta en la perspectiva político - ideológica de la campaña como un problema clave en el desarrollo nacional.

Analfabetismo y subdesarrollo se presentan íntimamente relacionados. El proyecto educativo destaca como la primera prioridad de la política educativa la necesidad de "erradicar el analfabetismo" basada en los datos del Censo de 1960 que informa sobre la existencia de un $8,6 \%$ de analfabetos. Como segunda prioridad se propone "disminuir el semianalfabetismo". Este concepto, en el documento del Plan Trienal ${ }^{3}$, abarca a las personas que no completaron la escolaridad primaria y que representa el $42 \%$ de la población mayor de 14 años.

Los documentos elaborados para la campaña proponían la vinculación directa entre el "Subdesarrollo en sus distintas manifestaciones" con el "problema del analfabetismo". Y establecía que sus causas son de índole política, económica y social, fundamentalmente en los países pertenecientes al tercer mundo".

Estas definiciones político- ideológicas no sólo se expresan en documentos generales de la campaña y de la política educativa, sino que son presentados en los manuales destinados a los alfabetizadores. En el manual "El Pueblo Educa al Pueblo" se hace referencia al analfabetismo como un problema que

[...] radica en la clase trabajadora y otros sectores oprimidos de las sociedades neocoloniales... por eso los movimientos de liberación del tercer mundo han integrado al objetivo general de sus planes de desarrollo económico-social, la exigencia de erradicar el analfabetismo y semianalfabetismo e incluso han iniciado esas acciones durante la lucha por su liberación. (1973, p. 5).

En tal sentido, Freire propone la alfabetización y la concientización como procesos que se dan al mismo tiempo en el conocimiento de su propia realidad y en la objetivación de las condiciones de explotación. Freire encuentra en esos procesos la clave de un "proceso pedagógico que puede llevar a las clases oprimidas a tomar conciencia de su propia situación" (1983, p. 46)

\footnotetext{
${ }^{3}$ El Plan Trienal era la formulación del plan de gobierno para todas las áreas de la política pública. Estaba destinado a desarrollarse entre los años 1974 y 1977.
} 
Se aprecia el vínculo establecido entre procesos revolucionarios y las acciones de liberación de la dependencia. En esos procesos la alfabetización se considera como una práctica social que puede aportar a tomar conciencia de la situación de explotación y por ello, preparar a los sujetos para superar las relaciones de dependencia entendida como dependencia económica, cultural científica y tecnológica. (Documento El Pueblo Educa al Pueblo)

En este sentido, una definición importante de la propuesta de la CREAR se puede apreciar en el posicionamiento acerca del analfabetismo, al señalar que el lenguaje es un “instrumento político de dominación”. Más concretamente, se plantea la concepción que a través de las imposiciones culturales también se impone un "lenguaje” (1974: 3)

Se enuncia que el analfabetismo es el resultado de estructuras sociales desiguales, provocadas por la política internacional. En esa perspectiva se analiza el lenguaje como una forma de “dominación". En este sentido, en este documento también se presenta el analfabetismo...y en ese marco proponen que las culturas "dominantes" de los países imperialistas y las culturas “dominadas" se expresan a través de formas del lenguaje. En este razonamiento revalorizan la importancia de las culturas populares y la alfabetización desde los valores y la cultura del pueblo.

Los discursos de los documentos analizados consideran que el impacto de las políticas imperialistas se constituye en causas del analfabetismo. Por ello, desde la CREAR se propone, como forma alternativa a esos modelos, la construcción de procesos de recuperación de lo nacional y de la producción del pueblo.

En este marco, se construye un universo de referencias compartidas que articula la educación y en particular la alfabetización, como camino para acompañar los procesos revolucionarios en cada país. En esta línea, uno de los entrevistados manifiesta que “...era la alfabetización era parte de un proceso de organización social más amplio...” (Entrevista Subcoordinador Nacional de la CREAR ${ }^{4}$ )

Otro entrevistado asocia la alfabetización con procesos de concientización y de organización para el cambio social:

La cuestión era hacer evolucionar la conciencia...nos estábamos preparando para algo...Prepararse para el estalle necesariamente pasaba por educar, promocionar...prever que al momento del estalle todos estuviéramos organizados, llevábamos crear un nivel de conciencia. (Entrevista Coordinador de Base $^{5}$ )

\footnotetext{
${ }^{4}$ El subcoordinador nacional era un cargo dentro de DINEA, en el ministerio de educación nacional, destinado a coordinar la logística del despliegue territorial de la campaña en las provincias.

5 Coordinador de Base representa en el marco de esta campaña al alfabetizador, sin embargo, el cambio de denominación responde a otra forma de asumir sus tareas en el marco de la inserción territorial y comunitaria.
} 
Los relatos del coordinador provincial coinciden con los anteriores testimonios en plantear la educación como una herramienta en el proceso de cambio social cuando sostiene que

[...] llevar la instrucción al pueblo más necesitado desde la premisa o convicción de que se educa para el desarrollo y el progreso del país, sin educación no se pueden cambiar los proyectos, los caminos de la historia. Eso era un poco lo que estaba en el trasfondo de todo esto no, pensar que el cambio social también requería la formación y la instrucción del pueblo en su conjunto" (Entrevista Coordinador Provincial ${ }^{6}$ )

En las manifestaciones de uno de los entrevistados es posible apreciar los sentidos que la alfabetización alcanza en la organización política de los grupos.

Devolver la palabra, que pueda participar en las reuniones de la capilla, en las reuniones del centro vecinal, el empezar a mirarse de otra forma...yo rescato de la CREAR esa movida cultural que fue armando..." (Entrevista Coordinador de Área, zona 14)

Los decires de los coordinadores de la campaña expresan la construcción/apropiación en sus prácticas de lo que Williams (2009) denomina las estructuras de sentimiento de una época. La revolución, la dependencia, la descolonización y el pueblo como sujeto político son ideas que articulan las lecturas sobre el cambio social en distintos planos.

La articulación entre formas de conciencia y cambio social no se reduce a la alfabetización en los discursos analizados. Muy por el contrario, esta intencionalidad de producir rupturas que se expresan en la revisión del marxismo, de las ciencias sociales, también se traduce en la creación artística como parte de una nueva estética.

Es en esa dinámica de repensar el orden social, que entran en escena distintas producciones artísticas orientadas por otro concepto acerca del sentido social de lo artístico y de los sujetos sociales a los que se dirigen. Aveiro sostiene que en este contexto

[...] aparece la nueva canción popular latinoamericana... que empiezan a sembrar a través de la canción las ideas socialistas y el ideal del hombre nuevo. También en otros campos como la literatura... con su literatura de denuncia y compromiso social. Otro de los fenómenos será el cine... que mostraban la realidad social... (2006. p. 109-110)

En esta producción donde lo estético y lo intelectual se articulan en un discurso de emancipación y liberación es importante destacar que también se constituyen como un espacio de disputa de sentidos sobre los debates del momento para generar legitimidad.

\footnotetext{
${ }^{6}$ En las provincias había dos referentes principales de la implementación de la campaña. Por un lado, estaba el coordinador organizativo, abocado a tareas fundamentalmente de logística de todas las acciones que implicaba el despliegue de la campaña. Por otro lado, estaba la coordinación de capacitación docente, que tenía tareas pedagógicas dentro de la estructura de la campaña.
} 
Estas ideas se manifiestan en el discurso político de Cámpora ${ }^{7}$ :

El intelectual, el científico, el escritor, el artista, conscientes de la función social que les cabe, deberán aplicar su genio al acrecentamiento de la cultura del pueblo y la liberación de la patria... No existe cambio educativo sin cambio social, pero, complementariamente, no existe consolidación de cambio social sin efectivización del cambio educativo. (1973. p. 149-150)

Los sentidos expresados en este fragmento del discurso de Cámpora, sintetizan la importancia otorgada a la educación en el proceso revolucionario y su sostenimiento y legitimación. Pero al mismo tiempo, manifiesta el aporte que debían hacer los intelectuales y los artistas en ese proceso, donde los sentidos sociales de su tarea se encuentran vinculados al pueblo.

La alfabetización como forma de "crear conciencia" en el proceso de cambio social que la izquierda del peronismo denominó la revolución cultural, se construye en torno a las formas de organización social.

\section{Método CREAR: los aportes de Freire y la Psicología social}

La alfabetización se sostuvo en la CREAR estrechamente vinculada a la concientización. Por ello interesa analizar en este apartado como se articuló la alfabetización y la concientización en la propuesta metodológica de la campaña.

Una forma de analizar la cultura escrita en las campañas de alfabetización, es el estudio de los métodos de alfabetización de adultos que proponen. No como una cuestión técnica. Sino que entendemos que el método representa la convergencia de múltiples dimensiones del proyecto educativo, donde se condensan los sentidos sociales de la educación, los fundamentos políticos ideológicos, los componentes pedagógicos, los sujetos, los contenidos. La articulación de todos estos elementos, no siempre se produce de manera explícita, ni siempre desde la objetivación coherente de quienes la formulan. La articulación de todos estos elementos supone posicionamientos y concepciones que, integrados de forma más o menos explícitas, expresan una síntesis de opciones (Edelstein; Rodríguez, 1974).

En el marco de los principios señalados, un aporte fundamental de la campaña fue organizar procesos de alfabetización situados en prácticas colectivas.

\footnotetext{
${ }^{7}$ Tras 18 años de prescripción del peronismo gana las elecciones Héctor Cámpora. Asume la presidencia el 25 mayo del 73 y renuncia en julio de 1973 cuando Perón expresa su voluntad de ser presidente. La cercanía de Cámpora con la izquierda del peronismo lo enfrentó con la derecha sindical. Esta propuesta educativa fue producto de este gobierno, aunque es implementado fundamentalmente a partir del 8 de septiembre de 1973, estando ya Perón en la presidencia. Se desarrolla durante el ejercicio en el Ministerio de Educación del Dr. Jorge Taiana.
} 
Desde los posicionamientos antes expuestos, la propuesta de alfabetización de la campaña combino el método de Paulo Freire (1965) con los aportes de la escuela de Psicología social (Pichón Riviere, 1985).

En primer lugar, el método de alfabetización tomaba los aportes de Freire en cuanto a la estructura metodológica: selección del universo vocabular, codificación de situaciones existenciales, procesos de reflexión hasta llegar a la escritura y la lectura.

En segundo lugar, se basa en la escuela argentina de psicología social que proponen una mirada sobre las tareas de los grupos. En esos procesos grupales y la tarea de alfabetización como organizador de la existencia de los grupos, hay un posicionamiento epistemológico acerca del aprendizaje y su posibilidad de transformación de la realidad. Esto es así, ya que los contenidos que trabajan los grupos tienen que partir de su propia experiencia para que, convertidos en objeto de conocimiento y de crítica, puedan ser transformados. Por estos posicionamientos, hay una convergencia epistemológica entre Freire y la psicología social.

Los aportes de la escuela de psicología social posibilitaron además el sentido colectivo de los procesos de aprendizaje y la organización en torno a una tarea a través de los grupos operativos de aprendizaje.

En este marco, resulta relevante analizar cómo se integran los momentos del método que propone Freire con las dinámicas de funcionamientos de los grupos operativos. Sostenemos que los puntos de convergencia de ambos enfoques son los contenidos y los procesos de reflexión con el objetivo de transformar la realidad.

En efecto, estas propuestas sostienen un posicionamiento similar en cuanto al proceso de conocimiento y del sentido transformador del mismo en la praxis de los sujetos. Tal como plantea Freire, las praxis se refieren a las acciones de los sujetos orientadas por finalidades que tienen sentido en un proceso histórico

[...] la interrelación entre la conciencia del proyecto propuesto y el proceso para alcanzarlo, es la base de la acción planificada del ser humano, la cual implica métodos, objetivos y opciones de valor. (FREIRE, 1983, p.18)

En tal sentido y de forma convergente con la psicología social, la actividad de los sujetos en el proceso de conocimiento es entendida como praxis en tanto opera sobre la realidad para transformarla (QUIROGA, 1984)

El contenido existencial como objeto de crítica y transformación por parte de los sujetos constituye en espacio de síntesis de estas propuestas como construcción metodológica. 
Nos detendremos particularmente, en los contenidos y los procesos de reflexión ya que en ellos se manifiestan los sentidos ideológicos, políticos y educativos de la CREAR donde genera una alternativa a las pedagógicas dominantes para la alfabetización de adultos.

Interesa señalar que cobran relevancia analítica los contenidos y los procesos de reflexión, ya que expresan los temas que fueron objeto de las formas de educación y el proceso de tratamiento de esos temas por parte de los grupos. En tal sentido, analizamos la propuesta de los documentos y los testimonios de los entrevistados para comprender el énfasis puesto en los procesos de reflexión de los grupos como una forma de concientizar.

Desde los posicionamientos teóricos sostenidos en el discurso de la campaña, entendemos que las situaciones existenciales y las palabras generadoras - en tanto contenidos de reflexión- no podían ser seleccionadas azarosamente. De esta manera se proponen, como palabras generadoras a nivel nacional, las palabras: Leche, Casa, Ropa, Pozo (o Pico), Familia (o hijo), Villa (o barrio), Vecino, Cooperativa, Escuela, dispensario, Ómnibus, Trabajo, Maquina, Patrón, Sueldo (jornal o quincena), Campesino (o peón -obrero) Sindicato, Delegado, Radio (o televisión), Futbol, Promesa, Vino, Compañero, Voto, Pueblo organizado, Gobierno, Latinoamérica unida o dominada. Luego, en cada provincia se podría agregar otras palabras generadoras que estuvieran vinculadas a las características regionales.

Tal como se expresa en el documento CREAR Propuesta Metodológica

La palabra generadora en este método, tiene como característica la de representar una situación clave que encierra una problemática socialmente significativa" (1973, p.8)

Los pasos del método CREAR suponían el relevamiento del universo vocabular, donde se integran situaciones existenciales recurrentes en los grupos. A esas situaciones se las asociaba con una palabra clave que se constituían en las palabras generadoras. Las situaciones eran usadas para la reflexión y las palabras generadoras para la lecto - escritura. Estos pasos constituyen un momento previo a la tarea de alfabetización. La misma se inicia con la presentación de la lámina u otro elemento. El objetivo es llegar al diálogo (El pueblo Educa al pueblo).

La presentación de la lámina se constituye en un "Disparador motivacional" en donde se presenta la palabra generadora codificada en una situación existencial.

Los documentos proponían la utilización de distintos recursos para la motivación de la tarea denominado "disparador direccional" como fotos o láminas. Luego, orientan los distintos momentos por los que atraviesa el grupo en la consecución de la tarea. En esos procesos establece las actividades del coordinador en dos niveles: centrando la discusión para 
orientarla hacia los objetivos $\mathrm{y}$, ordenando la dinámica del grupo para que todos puedan participar.

Tanto las palabras como las situaciones fueron elaboradas de acuerdo al criterio de que representen situaciones vividas por los trabajadores y el pueblo. Las palabras generadoras y las láminas/fotos donde se expresan las situaciones existenciales, son las bisagras en la articulación teórica metodológica ya que encierran los posicionamientos políticos e ideológicos en los que se proponen formar a los trabajadores y marginados.

Metodológicamente, en primer lugar, en los grupos se definen las palabras, ya sea las nacionales o las modificadas de acuerdo a las características del grupo. Una coordinadora relata que, en lugar de usar trabajo como palabra generadora, utilizaron "laburo" o "changa" ya que era la denominación que utilizan esos grupos.

En base a los principios teóricos y epistemológicos que se sostienen en los documentos sobre el proceso de conocimiento, se observa un importante peso de los contenidos a trabajar en estos espacios. Por ejemplo, en el documento Bases de la CREAR se plantea como objetivos

Informar sobre problemas locales, regionales y nacionales, así como sobre la política gubernamental en el control de la producción, distribución y precios de artículos de primera necesidad. Se trata de garantizar desde la educación, para el logro de los objetivos en el área social y económica, que los sectores mayoritarios de la Argentina lleguen a tener un nivel de conocimiento de aquellos problemas relativamente semejante - en lo esencial- al que tienen las minorías" (CREAR, 1973, p. 8)

El criterio que se estableció para la selección de la problemática a trabajar fue que se vinculara con las problemáticas habituales de estos grupos (El pueblo educa al pueblo, 1973)

Tal como se sostiene en el documento Cartilla Guía para la coordinación del proceso de reflexión

Si la reflexión no se basa en la realidad, será un mero ejercicio intelectual. Por lo tanto, es importante que el coordinador logre, que los participantes, expresen en el mejor grado de su posibilidad, como ven y cómo viven la situación. (1973, p..2)

Nuevamente aquí se expresa la concepción de conocimiento que, partiendo del análisis de las situaciones sociales de los grupos, de sus organizaciones y sus necesidades se convierten en objeto de crítica por parte de esos mismos grupos, lo que implica procesos de objetivación que permitirán su transformación.

La orientación desde esta perspectiva epistemológica se materializa en los documentos en unos pasos que permitieran que el grupo construya esos momentos. En el 
documento El pueblo Educa al pueblo se proponen "pasos metodológicos” que tienen por objetivo orientar la reflexión del grupo en los siguientes puntos: "Ver base para apreciar; Apreciar base para conocer; Conocer base para resolver; Resolver base para ejecutar" (El pueblo educa al pueblo, 1973:14). Estos puntos se presentan como bisagras entre los pasos metodológicos y el proyecto político del peronismo.

Siguiendo estos pasos, se puede observar en los materiales nacionales las pautas generales para la decodificación a partir de una serie de preguntas orientadoras que estaban destinadas a la formación de los alfabetizadores. La secuencia es la siguiente:

- $\quad$ En primer lugar, se observan preguntas que apuntan a la descripción de la lámina, la interpretación narrativa a partir de la construcción de una historia de lo que pasa en la lámina.

- Luego se apuntaba a una conceptualización de lo que ven a través de la elaboración de un título para lo que han podido observar.

- A continuación, se proponía la identificación del o los problemas y la jerarquización de los mismos. Además, se debía comparar ese o esos problemas con lo que le pasa al propio grupo y cómo le pasa. Se propone también comparar su grupo con otros grupos para ver si esa problemática aparece y cómo aparece.

- Identificadas las problemáticas y en qué sectores se presenta, se procede al análisis de causas, consecuencias y los sujetos responsables. En ese marco, se consideraba la política del gobierno para la solución de esos problemas y se indagaba si sabían cómo han solucionado esa problemática en otros espacios.

En algunos casos se puede apreciar, que además de presentar las políticas del gobierno cómo contenido de reflexión, se la compara con las políticas que llevó adelante en esa área la dictadura militar (1966-1972). Es el caso del documento Cuadernillo para el Coordinador de Base de la CREAR (1974) analiza cómo se fija el precio de la carne, presenta un cuadro comparativo entre la "política de la dictadura y la política del gobierno popular" (1974:27-28), propone además una campaña para controlar los precios de los productos de primera necesidad.

Los distintos documentos metodológicos elaborados para la campaña: El Pueblo Educa al Pueblo, la Cartilla Guía para coordinar el proceso de reflexión insisten en la importancia de la posición del coordinador como un sujeto clave en la orientación y la conducción del grupo hacia la meta o realización de la tarea como plantea la psicología social. En este paso es clave que el alfabetizador/coordinador haya sido aceptado por el grupo en relación al tema o problemas que identifica el grupo. 
De acuerdo a lo desarrollado, en estos pasos del método se condensan los sentidos sobre las prácticas sociales de los grupos de alfabetizandos, partiendo de la oralidad y de la construcción de narrativas interpretativas de los materiales educativos: las láminas y las fotos. En algunos casos, también ha sido a partir de materiales que aportan los alfabetizandos como revistas o libros con determinada información. Pero también se advierte el análisis de situaciones tradicionales recuperadas a través de la oralidad de los sujetos: la preparación de determinadas infusiones, rituales para curar determinadas enfermedades, conocimientos producto de la práctica de algún oficio, entre otros, según señalaron los coordinadores entrevistados.

En este sentido, el momento de la reflexión tiene una singular importancia, que se puede evidenciar tanto en los documentos, como en los testimonios de los entrevistados.

Devolver la palabra...devolver la palabra no solo significa dejarlos hablar... implica también el que pueda participar en las reuniones de la capilla, en las reuniones del centro vecinal, el empezar a mirarse de otra forma. (Entrevista Coordinadora de Base $\mathrm{B}^{\circ}$ Comercial)

Los decires de esta coordinadora expresan los sentidos de la alfabetización como una práctica social en tanto los usos de la escritura se insertan en otras prácticas sociales y tienen un sentido social en esos contextos. Pero además estarían expresando, la objetivación como sujetos sociales y posibilitando formas de participación en las instituciones de su barrio.

En tal sentido, el coordinador de área de la zona 14 manifiesta:

A mí me parece que lo más importante que quedó, no voy a decir que sepan leer y escribir, sumar y restar porque eso todos saben sobre todo cuando les pagan mal. Pero lo más importante que yo ví, es que la gente pudiera conversar, pudiera intercambiar respecto de su lugar. Que esa pelea de perros, que ella no me ha saludado, que yo tampoco la voy saludar, entonces, esa facilidad con que la gente se enoja y no puede conversar los problemas que le afligen a todos... o que voy a querer cordón cuneta yo sola, ella que se arregle. Ese tipo de cosas, permitía el método de Freire que la gente comenzase a hablar...(Entrevista Coordinador de área zona "14")

Los coordinadores entrevistados coinciden en señalar la importancia de que estos procesos de alfabetización pudieran aportar herramientas que posibiliten hablar sobre sus problemas y vincularse de otra forma con sus vecinos.

Una de las palabras generadoras que aparecen como significativas en el relato de dos coordinadores es la palabra vino. Una de las coordinadoras de base, que realizaba sus tareas en barrio Comercial de Córdoba recuerda el abordaje realizado con la lámina para la palabra generadora vino. 
Empezamos a armar, problematizando situaciones y yo nunca me voy a olvidar que la primera palabra de ese universo vocabulario que había que empezar a formar, problematizando cuestiones, fue la palabra VINO... La palabra, la primera que surgió fue esa. ¿Porque qué hacía la gente? Tomaban mucho... (Entrevista Coordinadora de Base $\mathrm{B}^{\circ}$ Comercial)

En el relato de la coordinadora se retoma un problema de esa comunidad y el trabajo con la misma a partir del vínculo con el médico del dispensario quien la ayudó en la tarea en el centro. Estos trabajos expresan las relaciones interinstitucionales que posibilitaba el desarrollo comunitario de la campaña y que aportan a una mirada integral de la alfabetización.

Otro de los coordinadores de base recuerda una situación, relatada por los alfabetizandos, relacionada con el alcoholismo como problema social. En este caso en la zona norte de la ciudad de Córdoba:

[...] porque había una señora Lidia, joven pero de esas mujeres jóvenes que parecen viejas arrugadas por la fuerza, el trabajo, la vida. Ella tenía una hija de 16 años que vivía en Los Cortaderos que se emborrachaba ella y el marido y para que no joda el niño le daban el biberón. Entonces todo conexo viste? a esa gran problemática que sigue siendo problemática en la zona de los cortaderos. Sigue actualmente. Y ahí trabajamos con el tema de la maternidad, la paternidad responsable. Con las palabras como para que lo entiendan. Los procesos de la lactancia, la importancia que tiene, los controles y todas esas cosas. Es decir, una palabrita que llenaba una amplitud de campos para trabajar que vos tenías que ir seleccionando..." (Entrevista coordinador de base zona 14)

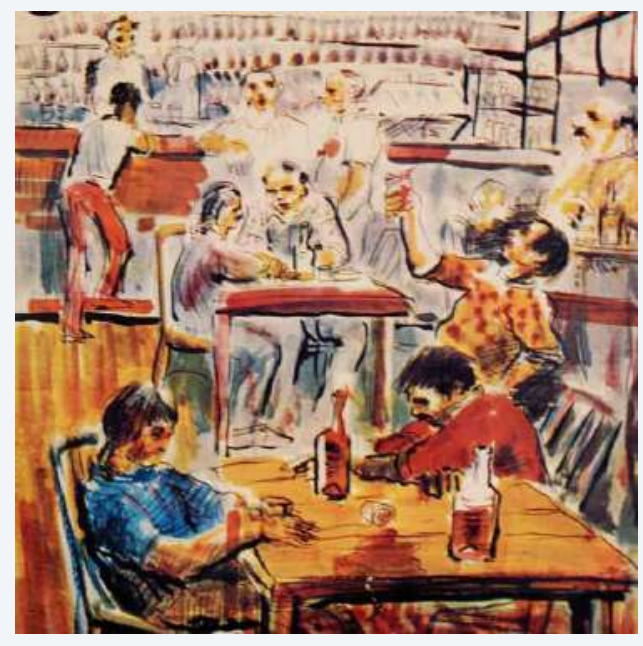

Lámina para la palabra generadora Vino. Extraída de la compilación de los materiales de la campaña reeditada en el año 2008, por el Ministerio de Educación de la Nación

En el manual se propone como objetivos de reflexión para la palabra vino los siguientes: 
"Palabra: VINO

1. Objetivos de la reflexión

a. Dialogar sobre el vino, como elemento incorporado a la vida social y como un complemento de la alimentación.

b. Discutir sobre el problema del alcoholismo:

- cómo se manifiesta en la comunidad del grupo alfabetizando;

- qué efectos produce en la salud del alcohólico

- qué efectos produce en la familia del alcohólico

- qué efectos produce en la comunidad

- qué efectos produce en el trabajo

c. Intercambiar opiniones sobre:

- las causas del alcoholismo

- las soluciones que se pueden dar a nivel de la comunidad organizada.

d. Conocer la política del Gobierno Popular frente a:

- el problema del alcoholismo

- la producción y comercialización del vino." (Método Crear. Material de apoyo, 2008. Pp. 121)

En el caso de la palabra vino se indica como objetivo: conocer la política del gobierno referida a la problemática del alcoholismo y a la producción y comercialización del vino. Propone además ese documento como contenido de reflexión, consultar los aspectos centrales del Plan Trienal. En ese sentido, resulta relevante que las políticas nacionales se constituyeran en contenidos de los procesos de reflexión por parte de los grupos.

El método de alfabetización es expresión del proyecto político, fundamentalmente del vínculo del peronismo de izquierda con el pueblo y su intento de organizar las bases se instala como forma de educar a las masas.

También resulta significativa en el recuerdo de una coordinadora, el tratamiento de la palabra agua ya que el acceso a la misma forma parte de una serie de problemas sanitarios en distintas comunidades. En muchos de estos espacios se observan canillas comunitarias compartidas por los vecinos:

Recuerdo una gran discusión que hubo con la palabra agua porque para muchas comunidades agua era el pico por donde salía el agua...cuál era la palabra para expresar la problemática del grupo... para que se pudieran organizar y reclamar... en esa época poco hablábamos de los derechos... con estos dos compañeros de ciencias de la información salíamos y tratábamos de encontrar situaciones que pudieran ser vinculadas con la palabra generadora que ya estaba fijada 


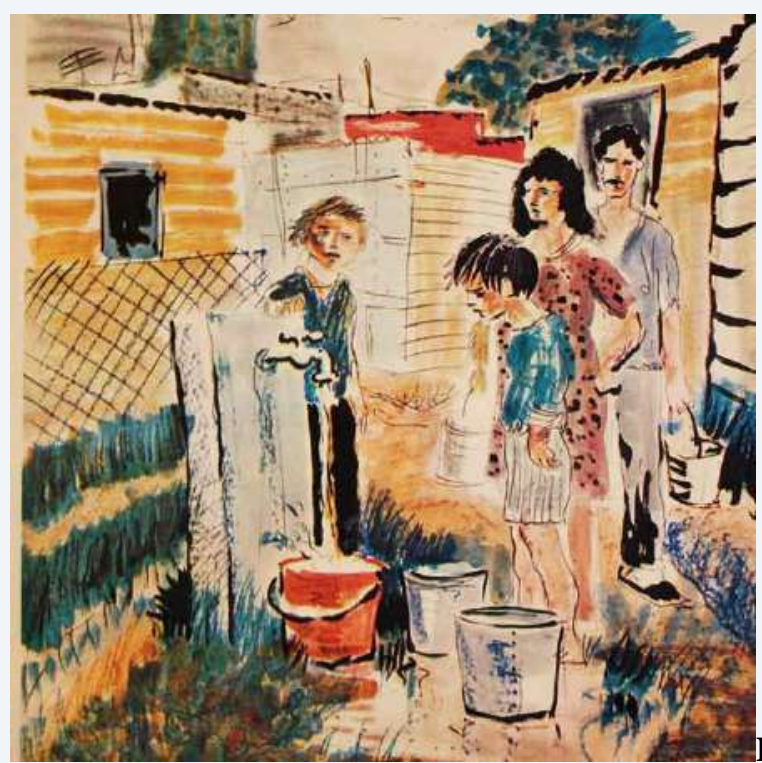

Lámina para la palabra generadora Agua. Extraída de la compilación de los materiales de la campaña reeditada en el año 2008, por el Ministerio de Educación de la Nación

Otro de los elementos importantes como contenidos de la reflexión es el encuadre de la problemática con el proyecto político del gobierno. Esto se expresa fundamentalmente en la recurrencia permanente al plan trienal y otras políticas relacionadas, por ejemplo, con el voto de la mujer. Es posible apreciar en el tratamiento de la palabra voto, que las políticas del gobierno se analizan en relación con la coyuntura, pero también en una perspectiva diacrónica, recuperando la historia del movimiento peronista.

Tanto los objetivos como los contenidos de las distintas palabras, presentan orientaciones que se proponen posibilitar rupturas con las situaciones asistencialistas y se refuerza la tarea de análisis de las causas de los problemas y la solución o soluciones que pueda construir ese grupo.

\section{Reflexiones finales}

En esta colaboración nos propusimos analizar los vínculos con la cultura escrita que se proponían desde una campaña nacional de alfabetización desarrollada en Argentina entre los años 1973 y 1975.

La pregunta inicial que propusimos para interpelar los sentidos de la cultura escrita fue ¿qué implican las propuestas masivas de alfabetización en cuanto a cómo se construyen las subjetividades con relación a la cultura escrita? 
En ese marco, situamos esa pregunta en los procesos de construcción hegemónica para entender los contextos de alfabetización y el desarrollo de las políticas en la educación de adultos como parte de la tarea educativa del estado.

Analizamos cómo la alfabetización fue entendida en la campaña como una herramienta de concientización en el proceso de construir legitimidad sobre un modelo de desarrollo en Argentina en un marco más amplio de disputa hegemónica en América Latina. En esa dinámica, la alfabetización era entendida, además, como una forma de apropiarse de un elemento de la cultura dominante - la lengua escrita - por parte de los oprimidos.

Desde las perspectivas desarrolladas sobre la cultura escrita, los contenidos con los cuales se iban a alfabetizar tenían que estar vinculados a la vida cotidiana de los sujetos y debían recuperar la cultura popular, entendida como la cultura producida por los sectores subalternos.

La psicología social y el planteo metodológico de alfabetización confluyeron en una propuesta donde el conocimiento cotidiano aparecía como una objetivación del lugar del sujeto en el proceso histórico social, a partir de la problematización de las condiciones materiales de existencia, entendiendo lo cotidiano como el espacio de reproducción social.

Además, los contenidos de la alfabetización que se expresan en el universo vocabular y en los materiales educativos muestran la focalización en determinadas prácticas sociales. Por ejemplo, el análisis de la palabra vino como producto social y el alcoholismo como problema que afecta a los trabajadores.

La alfabetización fue entendida como una forma de concientización, intentó superar el sentido mecanicista de decodificar un código, para formar en prácticas sociales de autogestión comunitaria.

"Devolver la palabra" esta frase que retomamos de uno de los coordinadores/alfabetizador, expresa desde nuestro análisis, los sentidos políticos pedagógicos que emergen de la aplicación de la propuesta metodológica de Freire y que orientó el accionar de los coordinadores. Es decir, la alfabetización se concibe no solo como la apropiación del código escrito, sino como un proceso de liberación.

\section{REFERENCIAS}

ANSALDI, Waldo; GIORDANO, Verónica. América Latina: la construcción del orden”. Tomo I. Editorial Ariel, 2012.

AVEIRO, Martín. La irrupción de la pedagogía de la liberación: un proyecto ético político de educación popular (Mendoza 1973). Miño y Dávila, 2006. 
DEVÉS VALDÉS, Eduardo. El pensamiento Latinoamericano en el siglo XX. Tomo II: desde la CEPAL al neoliberalismo (1950-1990). $2^{\circ}$ ed. Buenos Aires: Biblos, 2008.

EDELSTEIN, Gloria; RODRÍGUEZ Azuzena. El método: factor definitorio y unificador de la instrumentación didáctica. Revista de Ciencias de la Educación, Buenos Aires, Editorial Axis, IV (12), 1974. pp. 21-33. 1974.

FREIRE, Paulo. La educación como práctica de la libertad. Siglo XXI, 1965

FREIRE, Paulo. Acción cultural para la Libertad. Ed. Tierra Nueva, 1983.

GRAMSCI, Antonio. Notas sobre Maquiavelo, sobre la política y el estado moderno. Nueva Visión. Bs. As. Traducción de José Aricó. Ed. 2011.

GRAMSCI, Antonio. Los intelectuales y la organización de la cultura. Ed. Nueva Visión. Buenos Aires. 2006.

KALMAN, Judith. Discusiones conceptuales en el campo de la cultura escrita. Revista Iberoamericana de Educación. n. 45. p. 107-134. 2008.

KALMAN, Judith. Escribir en la plaza. Fondo de Cultura Económica. $1^{\circ}$ ed. En español. México. 2003.

KALMAN, Judith. Contexto de alfabetización. Texto basado en la conferencia magistral presentada el 8 de septiembre 2000, en Morelia, Michoacán, con motivo del Día Internacional de la Alfabetización. 2000.

LORENZATTI, María del Carmen. Conocimiento, prácticas sociales y usos escolares de cultura escrita de adultos de baja escolaridad”. Tesis de doctorado. FFyH. UNC. Córdoba, 2009.

PERESSON, Mario; CENDALES, Lola; MARIÑO, Germán. Educación Popular y alfabetización en América Latina. Dimensión Educativa. Bogotá. Colombia, 1983.

PICHÓN RIVIERE, E. Conversaciones con E. Pichón Riviere. Sobre el arte y la locura. Buenos Aires: Cinco, 1985

PINEAU, Pablo. ¿Qué es lo popular de la educación popular? Una aproximación histórica. En Elizalde, R. Ampudia, M. compiladores. (2008) "Movimientos sociales y educación. Teoría e historia de la Educación Popular en Argentina y América Latina". Buenos Aires: Buenos Libros, 2008.

PUIGGRÓS, Adriana; GÓMEZ, Marcela. Coord. Alternativas Pedagógicas. Sujetos y prospectiva de la Educación Latinoamericana.” Niño y Dávila. 2005.

QUIROGA, Ana. Matrices de aprendizaje. Constitución del sujeto en el proceso de conocimiento. Ana P. Quiroga. Edit. Cinco. Colección Apuntes. Buenos Aires. 1994. 
ROCKWELL, Elsie. Usos magisteriales de la lengua escrita. Nueva Antropología, vol. XII, núm. 42, julio, 1992, pp. 43-55 Asociación Nueva Antropología A.C. Distrito Federal, México. 1992.

RODRÍGUEZ, Lidia Mercedes. La especificidad en la educación de adultos: una perspectiva histórica en Argentina. En Revista Argentina de Educación. N. 13. p. 206-232. 1992.

WILLIAMS, Raymond. Marxismo y literatura. $1^{\text {o }}$ edición. Buenos Aires: Las Cuarenta, 1977.

\section{Documentos}

Bases para una política educativa del adulto. 1973. Ministerio de Cultura y Educación. Dirección Nacional de Educación del Adulto. Buenos Aires.

Bases de la Campaña de Reactivación Educativa del Adulto para la Reconstrucción. 1973. Ministerio de Cultura y Educación. Dirección Nacional de Educación del Adulto. Buenos Aires.

Ministerio de Educación (1973) DINEA El pueblo educa al pueblo. Buenos Aires. DINEA.

Ministerio de Educación (1973) DINEA. Método CREAR. Material de apoyo. Buenos Aires. DINEA. Plan Trienal para la Reconstrucción y la Liberación Nacional 1974-1977. Diciembre de 1973. Poder Ejecutivo Nacional. Bs. As. República Argentina.

\section{Entrevistas}

Miembro del equipo de lingüistas. Equipo central de DINEA nacional. Realizada el 11 de noviembre 2010 en el Instituto Nacional de Formación Docente. Buenos Aires.

Coordinador de área. Seccional 14. Zona Norte de Córdoba Capital. Realizada el 24 de marzo de 2010 en su casa, Córdoba Capital.

Coordinador de base. $\mathrm{B}^{\circ}$ Comercial Córdoba Capital. Realizada el 12 de noviembre de 2010 en su casa, Córdoba Capital.

Coordinador de base. Villa bustos. Córdoba Capital. Realizada el 23 de octubre de 2010 en su casa, Córdoba capital.

Coordinador de base. Seccional 14. Zona norte de la ciudad de Córdoba. Realizada el 15 de septiembre de 2010 en su casa, Córdoba capital.

\section{SOBRE A AUTORA:}

\section{Mariana A. Tosolini}

Doctora en Estudios Sociales de América Latina. Profesora y Licenciada en Ciencias de la Educación Docente en la Cátedra Historia Social de la Educación y en el Seminario Experiencias de Educación de Adultos en América Latina. Facultad de Filosofía y Humanidades. Universidad Nacional de Córdoba. Es investigadora en el Proyecto "Educación de jóvenes y adultos: sujetos, conocimientos y procesos de formación docente" con lugar de trabajo en el Área Educación del Centro de Investigaciones de la FFyH. Aprobado y Financiado por Secyt UNC. Res. No 313/2016. Y en el proyecto "Prácticas educativas con jóvenes y adultos: políticas, sujetos y conocimiento", con lugar de trabajo en el Área Educación del Centro de Investigaciones de la FFyH. Aprobado y Financiado por FONCYT. PICT-2016-2081. Res. No 285/2017. Ambos proyectos dirigidos por la Dra. María del Carmen Lorenzatti. E-mail: marianatosolini@gmail.com 